\title{
NUMERICAL SOLUTION OF NATURAL CONVECTIVE HEAT TRANSFER UNDER MAGNETIC FIELD EFFECT
}

\author{
Serpil ŞAHiN, ${ }^{*}$ Hüseyin DEMiR ${ }^{* *}$
}

\author{
${ }^{*}$ Faculty of Arts and Sciences, Department of Mathematics, Amasya University, 05000, Ipekköy, Amasya, Turkey \\ ${ }^{* *}$ Faculty of Arts and Sciences, Department of Mathematics, Ondokuz Mayıs University, 55200, Atakum, Samsun, Turkey \\ serpil.sahin@amasya.edu.tr, hdemir@omu.edu.tr
}

received 3 August 2018, revised 7 March 2019, accepted 13 March 2019

\begin{abstract}
In this study, non-Newtonian pseudoplastic fluid flow equations for 2-D steady, incompressible, the natural convective heat transfer are solved numerically by pseudo time derivative. The stability properties of natural convective heat transfer in an enclosed cavity region heated from below under magnetic field effect are investigated depending on the Rayleigh and Chandrasekhar numbers. Stability properties are studied, in particular, for the Rayleigh number from $\mathbf{1 0}^{\mathbf{4}}$ to $\mathbf{1 0}^{\mathbf{6}}$ and for the Chandrasekhar number 3,5 and 10 . As a result, when Rayleigh number is bigger than $\mathbf{1 0}^{\mathbf{6}}$ and Chandrasekhar number is bigger than $\mathbf{1 0}$, the instability occurs in the flow domain. The results obtained for natural convective heat transfer problem are shown in the figures for Newtonian and pseudoplastic fluids. Finally, the local Nusselt number is evaluated along the bottom wall.
\end{abstract}

Key words: Natural convective heat transfer, Rayleigh number, Chandrasekhar number, pseudo time derivative, Newtonian fluids, pseudoplastic fluids

\section{INTRODUCTION}

The stability and thermal convection in an enclosure have been subject of theoretical research since the time of Rayleigh (1916). Although the study of internal convection flows is more complicated than external flows, there has recently been a considerable interest in convection flows within closed geometries such as squares or rectangles. Thermal convection in enclosures occurs in many industrial applications such as building insulation, solar energy, and the electrical and nuclear industries. There are, for example, applications in heat transfer across double glazing windows and in sterilization of foods. Such flows are also of interest in geophysics so that several important examples can be applied in the circulation of the atmosphere and of magma in the Earth's upper mantle. Reviews of the literature for twodimensional free convection of rectangular enclosures have been reported by Gebhart et al. (1988) and Siginer and ValenzuelaRendon (1994).

The vertical cavity, with two vertical walls at different temperatures, is probably the most studied configuration due to its simplicity and importance. In a horizontal cavity, with heating from below, we have thermal instability. The other important phenomena in both cases are the buoyancy term involved in the flow equation. In thermal convection, the fluid density is no longer constant and depends on temperature in buoyancy term. Therefore, in such a region, the temperature of the cold wall $T_{0}$ is used as a reference to give the buoyancy term as $\rho \beta\left(T-T_{0}\right)$, where $\beta$ is the constant coefficient of thermal expansion, $\rho$ is the density of the fluid and $\mathrm{T}$ is the temperature (Tennehil et al., 1997).

Although many fluids encountered in various industries exhibit non-Newtonian behaviour, there are very few studies reported in the literature for thermal convection problems. Two surveys by Shenoy (1988) and Gebhart et al. (1988) mention only two studies on natural convection in enclosures.
Newtonian free convection has been extensively studied by Batchelor (1956), Elder (1965), Wilkes and Churchill (1966) and De Vahl Davis (1983). Emery et al. (1971) experimentally determined a correlation for the case of one wall at a constant temperature and a condition of constant heat flux at the other vertical wall for several pseudoplastic power law fluids.

Numerical methods are frequently used for two dimensional steady incompressible Newtonian flow problems. Due to the simplicity of cavity geometry, numerical methods can be applied very easily and effectively to this type of flow problems and results are very satisfactory.

For such studies, Benjamin and Denny (1979) have used a method that is relaxed by means of ADI methods using a nonuniform iteration parameter. Full converged solutions at Reynolds number up to $10^{4}$ with three different grid mesh sizes (maximum being $101 \times 101$ ) are generated in order to resolve basic questions on the nature of the flow and to explore convergence properties of the method.

Schereiber and Keller (1983) have introduced efficient and reliable numerical techniques of high-order accuracy for solving problems of steady viscous incompressible flow in the plane with these techniques, they obtain accurate solutions for the driven cavity. The numerical methods combine an efficient linear system solver and an adaptive Newton-like method for nonlinear systems. They have obtained solutions at Reynolds number 10000 on a $180 \times 180$.

Hou et al. (1995) have used Lattice Boltzmann Method for the simulation of the cavity flow. They have used $256 \times 256$ grid points and presented solutions for Reynolds number $\operatorname{Re} \leq 7500$.

Khader (2016) is devoted to introduce a numerical simulation with a theoretical study for the flow of a Newtonian fluid over an impermeable stretching sheet, which is embedded in a porous medium with a power law surface velocity and variable thickness in the presence of thermal radiation. 
Liao and Zhu (1996) have introduced a higher order stream function-vorticity boundary element method (BEM) formulation for the solution of Navier-Stokes equations. With this, they have presented solutions up to $\operatorname{Re}=10000$ with a grid mesh of $257 \times 257$.

Ghia et al. (1982) have applied a multi-grid strategy to the coupled strongly implicit method developed by Rubin and Khosla (1981). They have presented solutions for Reynolds number as high as $\operatorname{Re}=10000$ with meshes consisting of as many as $257 \times 257$ grid points.

Erturk et al. (2005) have introduced an effective numerical method for driven cavity flow by using stream function and vorticity formulation. Using a regular grid size of $601 \times 601$, they have solved the Navier-Stokes equations for $\mathrm{Re} \leq 21000$.

In the past decades, various numerical approaches have been used to solve magnetohydrodynamics (MHD) flows. A mixed finite element method has been discussed by Gunzburger et al. (1991). In Hasler et al. (2004), a weighted regularization approach has been applied to incompressible MHD system. Salah et al. (2001) have presented a Galerkin least-square method. Siddheswar et al. (2011) have introduced the higher order Rayleigh-Ritz (HORR) method, which is used to obtain the eigenvalue of the problem. Rudraiah et al. (1995) and Venkatachalappa et al. (2011) have used ADI (Alternating Direction Implicit) and SLOR (Successive Line Over Relaxation) method. Additionally, Amber and O'Donovan (2017) have presented two-dimensional simulations of natural convection driven by the absorption of nonuniform concentrated solar radiation in a molten binary salt-filled enclosure inclined at $0 \leq \varphi \leq 60$.

Recently, three classical iterative algorithms (Stokes iterative method, Newton iterative Method and Oseen iterative method) of solving 2D/3D Navier Stokes equations have been considered by $\mathrm{He}$ and $\mathrm{Li}$ (2009), Xu and $\mathrm{He}$ (2013).

Motivated by Erturk et al. (2005), this article is mainly focused on numerical solutions of natural convective heat transfer in an enclosed cavity heated from below.

\section{NUMERICAL METHOD}

The following figures schematically represent the flow in an enclosed cavity region heated from below under a magnetic field effect.

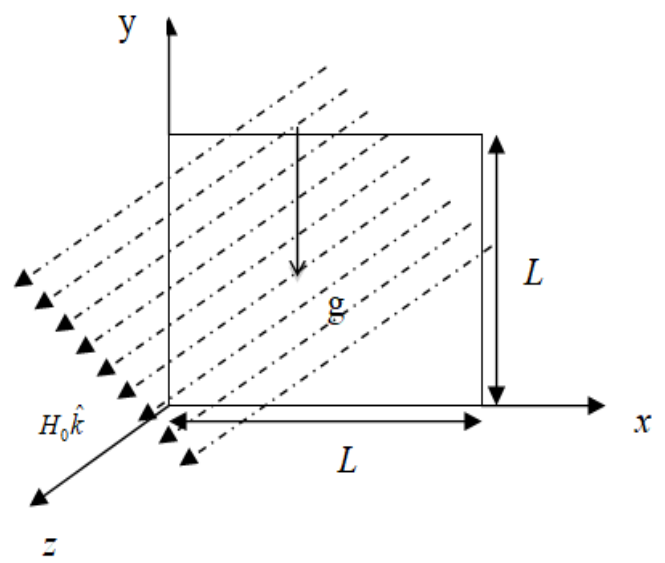

Fig. 1. Physical configuration and description of boundary condition
In Fig. 1, $\mathrm{L}$ is the characteristic length, $\mathrm{g}$ is the gravitational acceleration, $\mathrm{H}_{0}$ is the applied magnetic field and $\hat{\mathrm{k}}$ is a unit vector in the increasing direction of the space variable $\mathrm{z}$.

We use the stream function $(\psi)$, vorticity $(\omega)$ and temperature $(T)$ formulation of the steady-state incompressible pseudoplastic viscous fluid equations in the following form:

$u \frac{\partial \omega}{\partial x}+v \frac{\partial \omega}{\partial y}=\frac{1}{\operatorname{Re} \eta(q)}\left\{\frac{\partial}{\partial x}\left(\eta^{2}(q) \frac{\partial \omega}{\partial x}\right)\right.$

$\left.+\frac{\partial}{\partial y}\left(\eta^{2}(q) \frac{\partial \omega}{\partial y}\right)\right\}+\frac{1}{\operatorname{Re}}\left\{-4 \frac{\partial^{2} \psi}{\partial x \partial y} \frac{\partial^{2} \eta(q)}{\partial x \partial y}\right.$

$-\left(\frac{\partial^{2} \psi}{\partial y^{2}}-\frac{\partial^{2} \psi}{\partial x^{2}}\right)\left(\frac{\partial^{2} \eta(q)}{\partial y^{2}}-\frac{\partial^{2} \eta(q)}{\partial x^{2}}\right)$

$\left.+\frac{G r}{\operatorname{Re}} \frac{\partial T}{\partial x}-Q \omega\right\}$

$\frac{\partial^{2} \psi}{\partial x^{2}}+\frac{\partial^{2} \psi}{\partial y^{2}}=-\omega$

$u \frac{\partial T}{\partial x}+v \frac{\partial T}{\partial y}=\frac{1}{\operatorname{PrRe}} \nabla^{2} T$

$u=\frac{\partial \psi}{\partial y}, v=-\frac{\partial \psi}{\partial x}$

where, $\operatorname{Re}=\frac{\rho \mathrm{VL}}{\eta(0)}$ is the Reynolds number, $\mathrm{Gr}=\frac{\mathrm{g} \beta\left(\mathrm{T}_{1}-\mathrm{T}_{0}\right) \mathrm{L}^{3}}{v^{2}}$ is the Grashof number, $\operatorname{Pr}=\frac{c \eta(0)}{\mathrm{k}}$ is the Prandtl number, $Q=\frac{\mu_{\mathrm{m}}^{2} \sigma \mathrm{H}_{0}^{2} \mathrm{~L}}{u \rho}$ is the Chandrasekhar number, $q$ is the shear rate, $\eta(q)$ is the viscosity, $x$ and $y$ are the Cartesian coordinates, $\mu_{m}$ is the magnetic permeability, $\sigma$ is the surface tension gradient, $u$ is the velocity component in the $\mathrm{x}$-axis direction and $\rho$ is the density. Also, the Rayleigh number can be written as the product of the Grashof number, which describes the relationship between buoyancy and viscosity within a fluid, and the Prandtl number, which describes the relationship between momentum diffusivity and thermal diffusivity, that is, $\mathrm{Ra}=\mathrm{Gr}$. Pr. Finally, the Cross model is used in this work for the modelling of the viscosity function and this has the following form:

$\eta(q)=\eta(\infty)+\frac{(\eta(0)-\eta(\infty))}{1+(\lambda q)^{1-n}}$.

In this Cross Model, $\eta(\infty)$ represents the infinite shear viscosity for very large deformation rates and $\eta(0)$ represents the zero shear rate viscosity for very small rates of shear. Assuming $n=0.5, \lambda=1, \eta(0)=1$ and $0 \leq \eta(\infty) \leq 1$, we obtain shear-thinning or the so-called pseudoplastic behaviour.

The first order pseudo time derivatives are now introduced so that the equations (1), (2) and (3) take the following form:

$$
\begin{aligned}
& \frac{\partial \omega}{\partial t}=-\frac{\partial \psi}{\partial y} \frac{\partial \omega}{\partial x}+\frac{\partial \psi}{\partial x} \frac{\partial \omega}{\partial y}+\frac{1}{\operatorname{Re} \eta(q)}\left\{\frac{\partial}{\partial x}\left(\eta^{2}(q) \frac{\partial \omega}{\partial x}\right)\right. \\
& \left.+\frac{\partial}{\partial y}\left(\eta^{2}(q) \frac{\partial \omega}{\partial y}\right)\right\}+\frac{1}{\operatorname{Re}}\left\{-4 \frac{\partial^{2} \psi}{\partial x \partial y} \frac{\partial^{2} \eta(q)}{\partial x \partial y}\right. \\
& \left.-\left(\frac{\partial^{2} \psi}{\partial y^{2}}-\frac{\partial^{2} \psi}{\partial x^{2}}\right)\left(\frac{\partial^{2} \eta(q)}{\partial y^{2}}-\frac{\partial^{2} \eta(q)}{\partial x^{2}}\right)+\frac{G r}{\operatorname{Re}} \frac{\partial T}{\partial x}-Q \omega\right\} \\
& \frac{\partial \psi}{\partial t}=\frac{\partial^{2} \psi}{\partial x^{2}}+\frac{\partial^{2} \psi}{\partial y^{2}}+\omega \\
& \frac{\partial T}{\partial t}=-\frac{\partial \psi}{\partial y} \frac{\partial T}{\partial x}+\frac{\partial \psi}{\partial x} \frac{\partial T}{\partial y}+\frac{1}{\operatorname{PrRe}} \nabla^{2} T
\end{aligned}
$$


Using forward difference approximation for the time derivatives in equations (5), (6) and (7), we obtain the following equations (Smith, 1978):

$$
\begin{aligned}
& \left(1-\frac{\Delta t}{\operatorname{Re}} \eta^{n} \frac{\partial^{2}}{\partial x^{2}}+\Delta t\left(\frac{\partial \psi}{\partial y}\right)^{n} \frac{\partial}{\partial x}-2 \frac{\Delta t}{\operatorname{Re}}\left(\frac{\partial \eta}{\partial x}\right)^{n} \frac{\partial}{\partial x}\right) \\
& \times\left(1-\frac{\Delta t}{\operatorname{Re}} \eta^{n} \frac{\partial^{2}}{\partial y^{2}}-\Delta t\left(\frac{\partial \psi}{\partial x}\right)^{n} \frac{\partial}{\partial y}-2 \frac{\Delta t}{\operatorname{Re}}\left(\frac{\partial \eta}{\partial y}\right)^{n} \frac{\partial}{\partial y}\right) \omega^{n+1} \\
& =\omega^{n}+\frac{\Delta t}{\operatorname{Re}}\left\{-4\left(\frac{\partial^{2} \psi}{\partial x \partial y}\right)^{n}\left(\frac{\partial^{2} \eta}{\partial x \partial y}\right)^{n}-\left[\left(\frac{\partial^{2} \psi}{\partial y^{2}}\right)^{n}-\left(\frac{\partial^{2} \psi}{\partial x^{2}}\right)^{n}\right]\right. \\
& \left.\times\left[\left(\frac{\partial^{2} \eta}{\partial y^{2}}\right)^{n}-\left(\frac{\partial^{2} \eta}{\partial x^{2}}\right)^{n}\right]+\frac{G r}{\operatorname{Re}}\left(\frac{\partial T}{\partial x}\right)^{n}-Q \omega^{n}\right\} \\
& \left(1-\Delta t \frac{\partial^{2}}{\partial x^{2}}\right)\left(1-\Delta t \frac{\partial^{2}}{\partial y^{2}}\right) \psi^{n+1}=\psi^{n}+\Delta t \omega^{n} \\
& \left(1-\frac{\Delta t}{\operatorname{Pr} \operatorname{Re}} \frac{\partial^{2}}{\partial x^{2}}+\Delta t\left(\frac{\partial \psi}{\partial y}\right)^{n} \frac{\partial}{\partial x}\right)\left(1-\frac{\Delta t}{\operatorname{PrRe}} \frac{\partial^{2}}{\partial y^{2}}\right. \\
& \left.-\Delta t\left(\frac{\partial \psi}{\partial x}\right)^{n} \frac{\partial}{\partial y}\right) T^{n+1}=T^{n}
\end{aligned}
$$

In the event of reaching a steady state, we have:

$$
\psi^{n+1}=\psi^{n},
$$

$\omega^{n+1}=\omega^{n}$,

and

$T^{n+1}=T^{n}$.

Using this result in the right hand side of equations (8), (9) and (10), we may write:

$$
\begin{aligned}
& \left(1-\frac{\Delta t}{\operatorname{Re}} \eta^{n} \frac{\partial^{2}}{\partial x^{2}}+\Delta t\left(\frac{\partial \psi}{\partial y}\right)^{n} \frac{\partial}{\partial x}-2 \frac{\Delta t}{\operatorname{Re}}\left(\frac{\partial \eta}{\partial x}\right)^{n} \frac{\partial}{\partial x}\right) \\
& \times\left(1-\frac{\Delta t}{\operatorname{Re}} \eta^{n} \frac{\partial^{2}}{\partial y^{2}}-\Delta t\left(\frac{\partial \psi}{\partial x}\right)^{n} \frac{\partial}{\partial y}-2 \frac{\Delta t}{\operatorname{Re}}\left(\frac{\partial \eta}{\partial y}\right)^{n} \frac{\partial}{\partial y}\right) \omega^{n+1} \\
& =\omega^{n}+\frac{\Delta t}{\operatorname{Re}}\left\{-4\left(\frac{\partial^{2} \psi}{\partial x \partial y}\right)^{n}\left(\frac{\partial^{2} \eta}{\partial x \partial y}\right)^{n}-\left[\left(\frac{\partial^{2} \psi}{\partial y^{2}}\right)^{n}-\left(\frac{\partial^{2} \psi}{\partial x^{2}}\right)^{n}\right]\right. \\
& \left.\times\left[\left(\frac{\partial^{2} \eta}{\partial y^{2}}\right)^{n}-\left(\frac{\partial^{2} \eta}{\partial x^{2}}\right)^{n}\right]+\frac{G r}{\operatorname{Re}}\left(\frac{\partial T}{\partial x}\right)^{n}-Q \omega^{n}\right\}+\left(\frac{\Delta t}{\operatorname{Re}} \eta^{n} \frac{\partial^{2}}{\partial x^{2}}\right. \\
& \left.-\Delta t\left(\frac{\partial \psi}{\partial y}\right)^{n} \frac{\partial}{\partial x}+2 \frac{\Delta t}{\operatorname{Re}}\left(\frac{\partial \eta}{\partial x}\right)^{n} \frac{\partial}{\partial x}\right) \times\left(\frac{\Delta t}{\operatorname{Re}} \eta^{n} \frac{\partial^{2}}{\partial y^{2}}\right. \\
& \left.+\Delta t\left(\frac{\partial \psi}{\partial x}\right)^{n} \frac{\partial}{\partial y}+2 \frac{\Delta t}{\operatorname{Re}}\left(\frac{\partial \eta}{\partial y}\right)^{n} \frac{\partial}{\partial y}\right) \omega^{n} \\
& \left(1-\Delta t \frac{\partial^{2}}{\partial x^{2}}\right)\left(1-\Delta t \frac{\partial^{2}}{\partial y^{2}}\right) \psi^{n+1}=\psi^{n}+\Delta t \omega^{n} \\
& +\left(\Delta t \frac{\partial^{2}}{\partial x^{2}}\right)\left(\Delta t \frac{\partial^{2}}{\partial y^{2}}\right) \psi^{n} \\
& \left(1-\frac{\Delta t}{\operatorname{PrRe}} \frac{\partial^{2}}{\partial x^{2}}+\Delta t\left(\frac{\partial \psi}{\partial y}\right)^{n} \frac{\partial}{\partial x}\right)\left(1-\frac{\Delta t}{\operatorname{PrRe}} \frac{\partial^{2}}{\partial y^{2}}\right. \\
& \left.-\Delta t\left(\frac{\partial \psi}{\partial x}\right)^{n} \frac{\partial}{\partial y}\right) T^{n+1}=T^{n}+\left(\frac{\Delta t}{\operatorname{PrRe}} \frac{\partial^{2}}{\partial x^{2}}\right. \\
& \left.-\Delta t\left(\frac{\partial \psi}{\partial y}\right)^{n} \frac{\partial}{\partial x}\right) \times\left(\frac{\Delta t}{\operatorname{PrRe}} \frac{\partial^{2}}{\partial y^{2}}+\Delta t\left(\frac{\partial \psi}{\partial x}\right)^{n} \frac{\partial}{\partial y}\right) T^{n} \\
& +\operatorname{The~soluton~method~for~the~equations~}(14),(15) \text { and }
\end{aligned}
$$

The solution method for the equations (14), (15) and (16) involves a two-level updating. First, the stream function equation is solved. For equation (15), the variable $f$ is introduced such that:

$$
\left(1-\Delta t \frac{\partial^{2}}{\partial y^{2}}\right) \psi^{n+1}=f,
$$

and:

$$
\left(1-\Delta t \frac{\partial^{2}}{\partial x^{2}}\right) f=\psi^{n}+\Delta t \omega^{n}+\left(\Delta t \frac{\partial^{2}}{\partial x^{2}}\right)\left(\Delta t \frac{\partial^{2}}{\partial y^{2}}\right) \psi^{n}
$$

In equation (18), $f$ is the only unknown and is first solved at each grid point. Following this, the stream function variable $(\psi)$ is advanced into the new time level using equation (17). Next, the vorticity and energy equations are solved in a similar fashion.

The local Nusselt number $(\mathrm{Nu})$ has been evaluated along the bottom wall with the expression:

$$
N u=\frac{\partial T}{\partial y} \mid \quad y=0
$$

where, $T$ is the dimensionless temperature.

\section{RESULTS AND DISCUSSION}

We use the symmetry for , $\psi$ and $T$ at spurious points outside the boundaries. On the boundaries, the values of vorticity are chosen from the nine-point compact finite difference.

\section{Left Boundary Right Boundary}

$\psi_{-1, j}=\psi_{1, j}, \psi_{0, j}=0 \psi_{N+1, j}=\psi_{N-1, j}, \psi_{N, j}=0$

$u=v=0, \frac{\partial T}{\partial x}=0 \quad u=v=0, \frac{\partial T}{\partial x}=0$

$\omega_{0, j}=-\left(\frac{\partial^{2} \psi}{\partial x^{2}}\right)_{0, j} \omega_{N, j}=-\left(\frac{\partial^{2} \psi}{\partial x^{2}}\right)_{N, j}$

\section{Bottom Boundary Top Boundary}

$\psi_{i,-1}=\psi_{i, 1}, \psi_{i, 0}=0 \psi_{i, N+1}=\psi_{i, N-1}, \psi_{i, N}=0$

$u=v=0, T=T_{0} \quad u=v=0, T=T_{1}$

$\omega_{i, 0}=-\left(\frac{\partial^{2} \psi}{\partial y^{2}}\right)_{i, 0} \omega_{i, N}=-\left(\frac{\partial^{2} \psi}{\partial y^{2}}\right)_{i, N}$

We use the same algorithm with Erturk et al. (2001), but our convergence criterion is different. The convergence criterion that was used was based on the relative-error criterion (Demir, 2005):

$\max \left\{\frac{\left|\psi^{n+1}-\psi^{n}\right|}{1+\left|\psi^{n}\right|}\right\}<10^{-6}, \forall n$,

$\max \left\{\frac{\left|\omega^{n+1}-\omega^{n}\right|}{1+\left|\omega^{n}\right|}\right\}<10^{-6}, \forall n$,

$\max \left\{\frac{\left|T^{n+1}-T^{n}\right|}{1+\left|T^{n}\right|}\right\}<10^{-10}, \forall n$.

Tab. 1 tabulates the maximum stream function value, the vorticity value at the centre of the primary vortex and also the centre location of the primary vortex for Newtonian fluid at $\mathrm{Re}=1000$ along with similar results found in the literature are compared. In Tab. 1, among the most significant results, Erturk et al. (2005) have solved the cavity flow on three different grid meshes $(401 \times 401,513 \times 513,601 \times 601)$ for $\operatorname{Re}=1000$.

Looking back to Tab. 1, for $\mathrm{Re}=1000$, our results are in very good agreement with the results of Schreiber and Keller (1983), Hou et al. (1995) and Erturk et al. (2005). From all these 
comparisons, we can conclude that even for $\operatorname{Re}=1000$, higher order approximations together with the use of fine grids are necessary for accuracy.

Stream and temperature lines corresponding to $Q=0$ and $\mathrm{Q}=5, \mathrm{Ra}=10^{4}, \mathrm{Ra}=10^{5}$ and $\mathrm{Ra}=10^{6}$ are given for Newtonian, pseudoplastic fluids and pseudoplastic fluids under the magnetic field effect in the following figures, respectively. Also, all the figures are presented in xy plane.

Tab. 1. Comparison of the properties of the primary vortex; the maximum stream function value, the vorticity value and the location of the centre, for Newtonian fluid at $\mathrm{Re}=1000$

\begin{tabular}{|c|c|c|c|c|c|}
\hline Reference & Grid & $\psi$ & $\omega$ & $x$ & $y$ \\
\hline & $401 \times 401$ & 0.118585 & 2.062761 & 0.5300 & 0.5650 \\
\cline { 2 - 6 } $\begin{array}{c}\text { Erturk et } \\
\text { al. (2005) }\end{array}$ & $513 \times 513$ & 0.118722 & 2.064765 & 0.5313 & 0.5645 \\
\cline { 2 - 6 } & $601 \times 601$ & 0.118781 & 2.065530 & 0.5300 & 0.5650 \\
\hline
\end{tabular}

\begin{tabular}{|c|c|c|c|c|c|}
\hline $\begin{array}{c}\text { Schreiber } \\
\text { and Keller } \\
(1983)\end{array}$ & $121 \times 121$ & 0.11492 & 2.0112 & - & - \\
\cline { 2 - 6 } & $141 \times 141$ & 0.11603 & 2.0268 & 0.52857 & 0.56429 \\
\hline $\begin{array}{c}\text { Ghia et al. } \\
(1982)\end{array}$ & $129 \times 129$ & 0.117929 & 2.04968 & 0.5313 & 0.5625 \\
\hline $\begin{array}{c}\text { Hou et al. } \\
(1995)\end{array}$ & $256 \times 256$ & 0.1178 & 2.0760 & 0.5333 & 0.5647 \\
\hline $\begin{array}{c}\text { Liao et al. } \\
(1996)\end{array}$ & $129 \times 129$ & 0.1160 & 2.0234 & 0.5313 & 0.5625 \\
\hline $\begin{array}{c}\text { Benjamin } \\
\text { et al. } \\
(1979)\end{array}$ & $101 \times 101$ & 0.1175 & 2.044 & - & - \\
\hline \begin{tabular}{c} 
Present \\
\cline { 2 - 6 }
\end{tabular} & $256 \times 256$ & 0.118182 & 2.05677 & 0.5313 & 0.5664 \\
\cline { 2 - 6 } & $401 \times 401$ & 0.118626 & 2.06322 & 0.5312 & 0.5661 \\
\hline
\end{tabular}

(a) $128 \times 128, R a=10^{4}, Q=0$

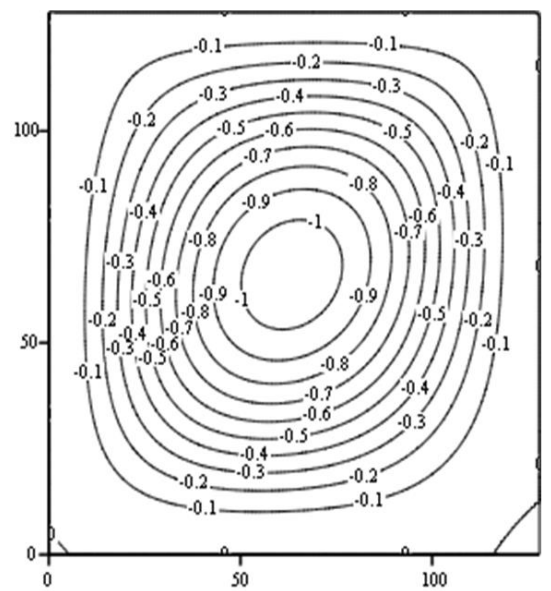

(b) $128 \times 128, R a=10^{4}, Q=0$

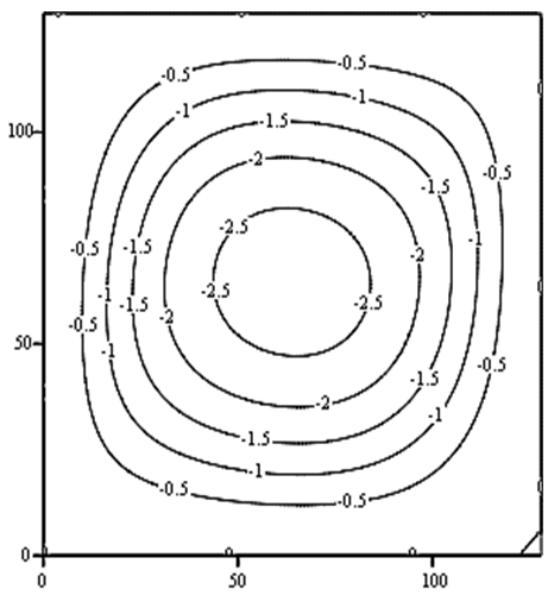

(c) $128 \times 128, \mathrm{Ra}=10^{4}, Q=5$

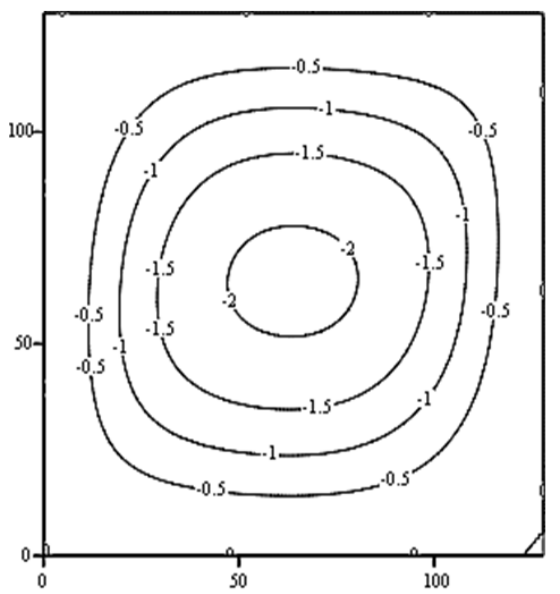

Fig. 2. Streamline contours for (a) Newtonian, (b) pseudoplastic fluids and (c) pseudoplastic fluids under the magnetic field effect at $R a=10^{4}$, respectively

(a) $128 \times 128, R a=10^{4}, Q=0$

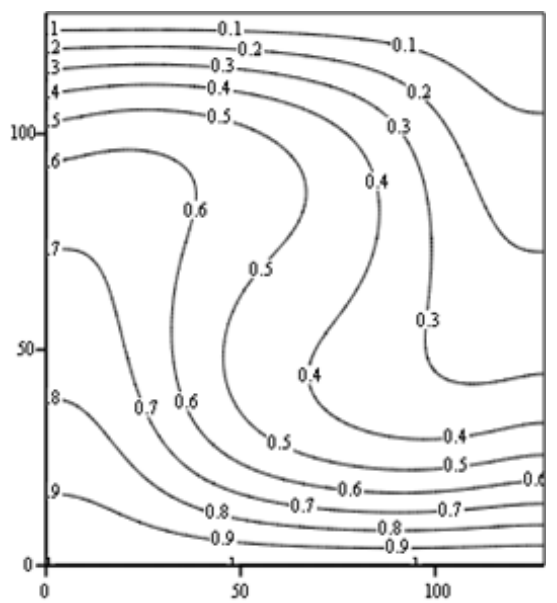

(b) $128 \times 128, R a=10^{4}, Q=0$

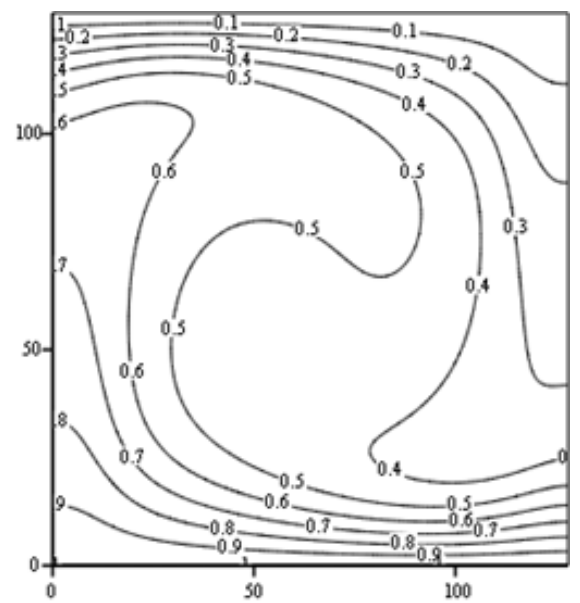

(c) $128 \times 128, R a=10^{4}, Q=5$

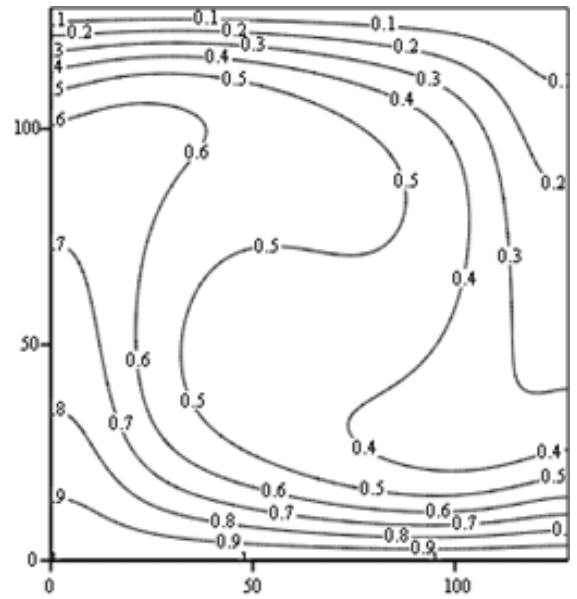

Fig. 3. Temperature contours for (a) Newtonian, (b) pseudoplastic fluids and (c) pseudoplastic fluids under the magnetic field effect at $R a=10^{4}$, respectively 
(a) $128 \times 128, R a=10^{5}, Q=0$

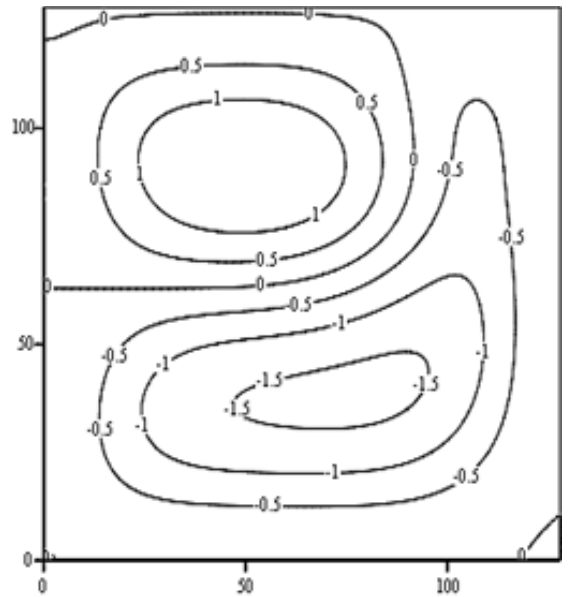

(b) $128 \times 128, R a=10^{5}, Q=0$

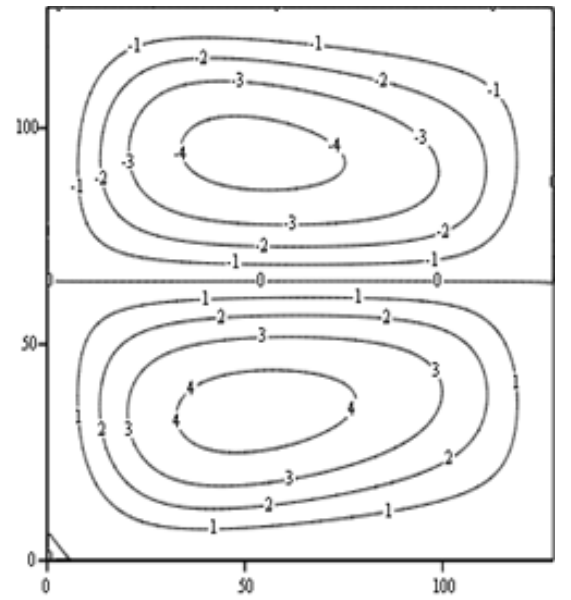

(c) $128 \times 128, R a=10^{5}, Q=5$

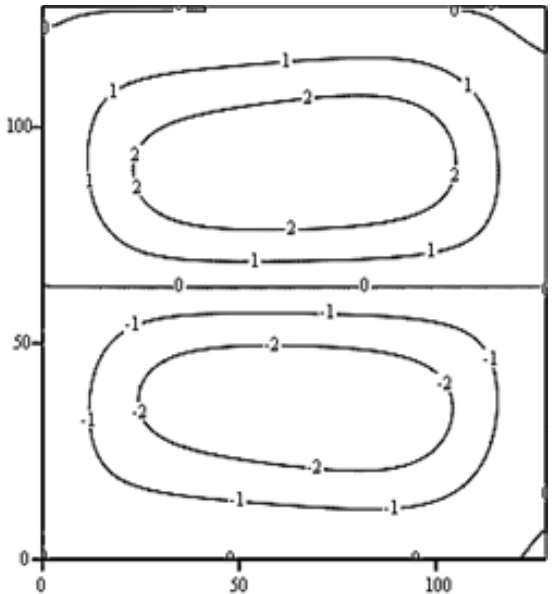

Fig. 4. Streamline contours for (a) Newtonian, (b) pseudoplastic fluids and (c) pseudoplastic fluids under the magnetic field effect at $R a=10^{5}$, respectively

(a) $128 \times 128, R a=10^{5}, Q=0$

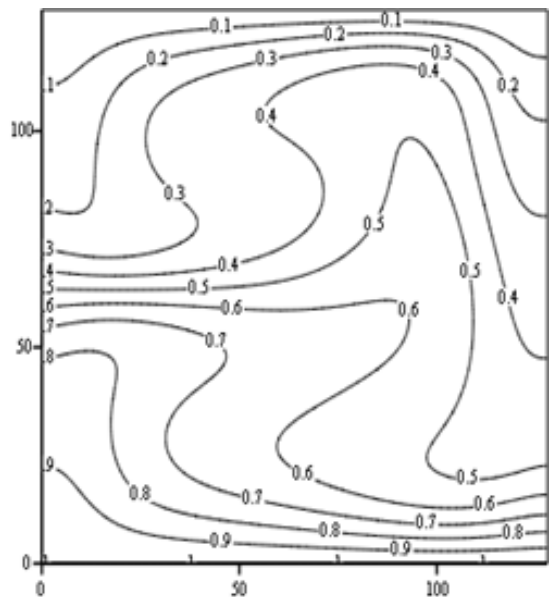

(b) $128 \times 128, R a=10^{5}, Q=0$

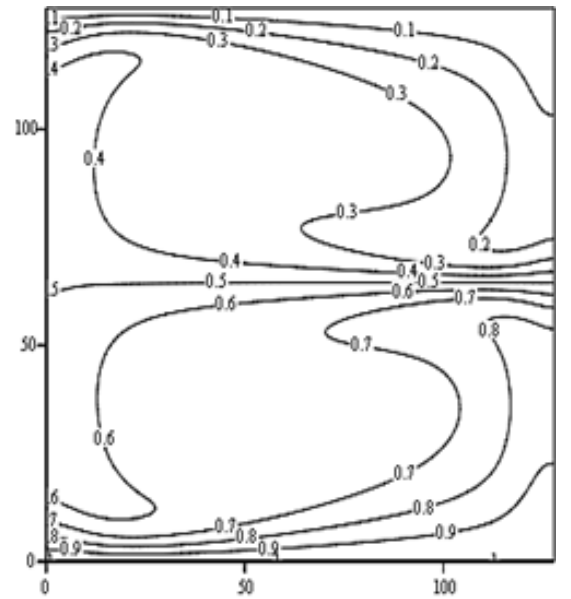

(c) $128 \times 128, R a=10^{5}, Q=5$

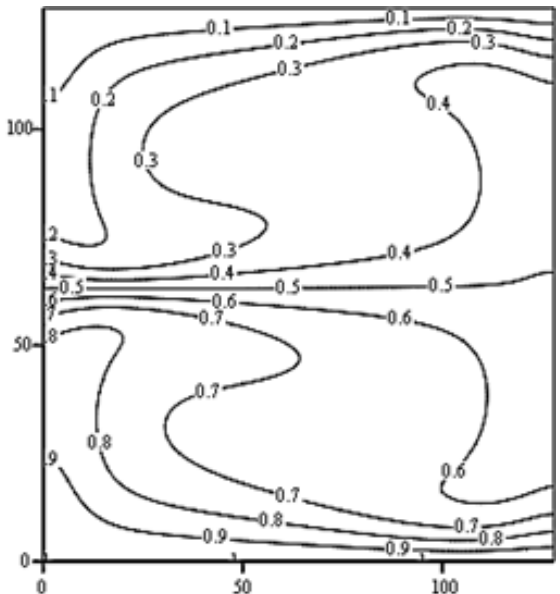

Fig. 5. Temperature contours for (a) Newtonian, (b) pseudoplastic fluids and (c) pseudoplastic fluids under the magnetic field effect at $R a=10^{5}$, respectively

(a) $128 \times 128, R a=10^{6}, Q=0$

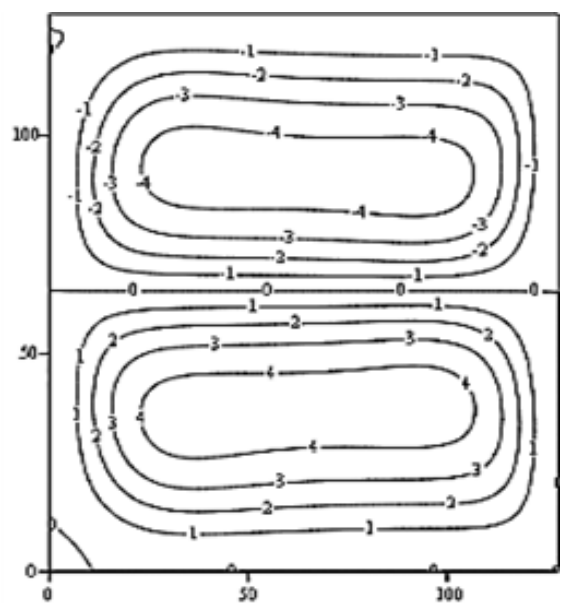

(b) $128 \times 128, R a=10^{6}, Q=0$

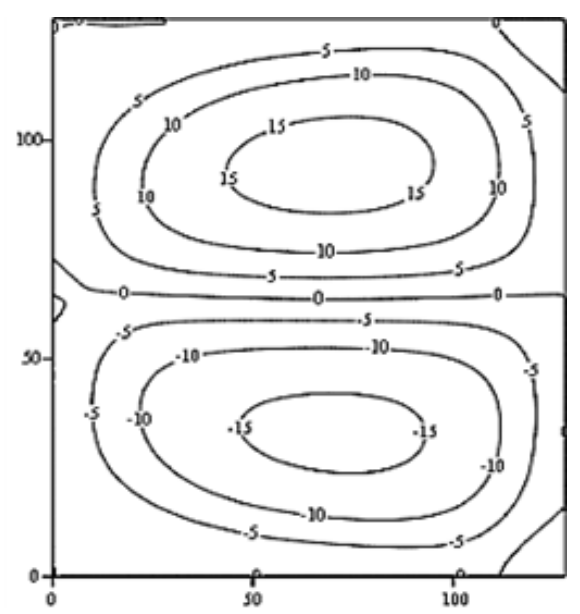

(c) $128 \times 128, R a=10^{6}, Q=5$

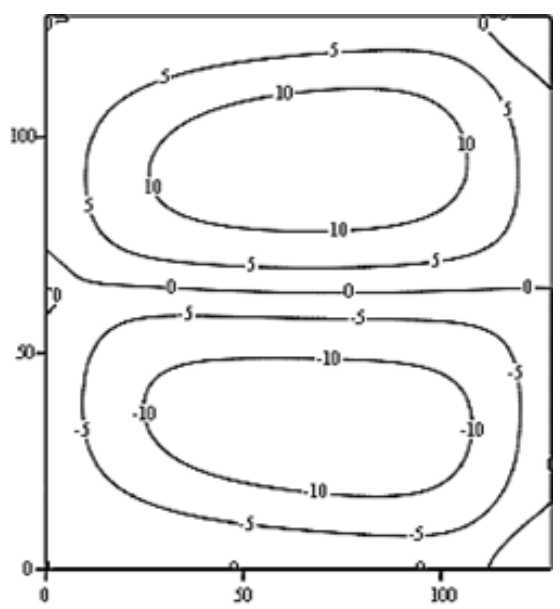

Fig. 6. Streamline contours for (a) Newtonian, (b) pseudoplastic fluids and (c) pseudoplastic fluids under the magnetic field effect at $R a=10^{6}$, respectively 
(a) $128 \times 128, R a=10^{6}, Q=0$

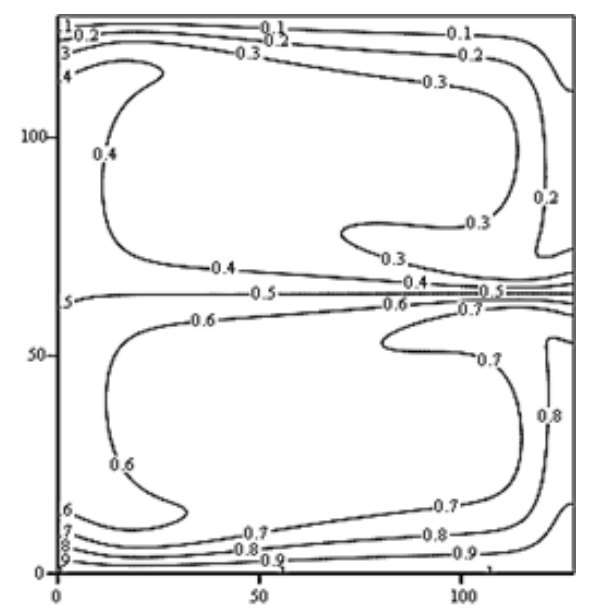

(b) $128 \times 128, R a=10^{6}, Q=0$

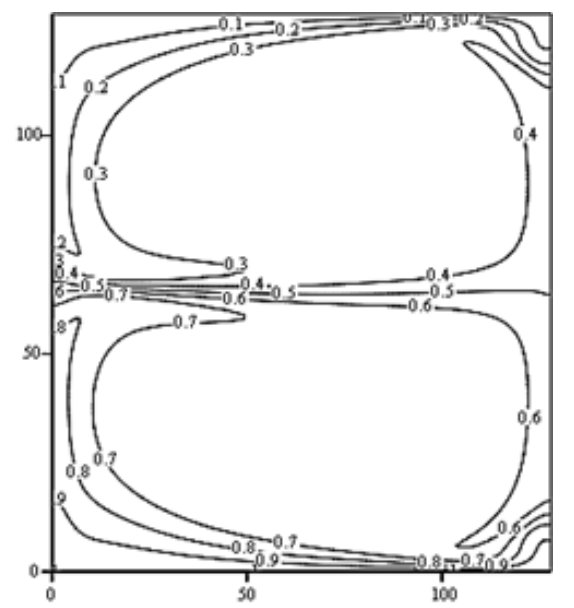

(c) $128 \times 128, R a=10^{6}, Q=5$

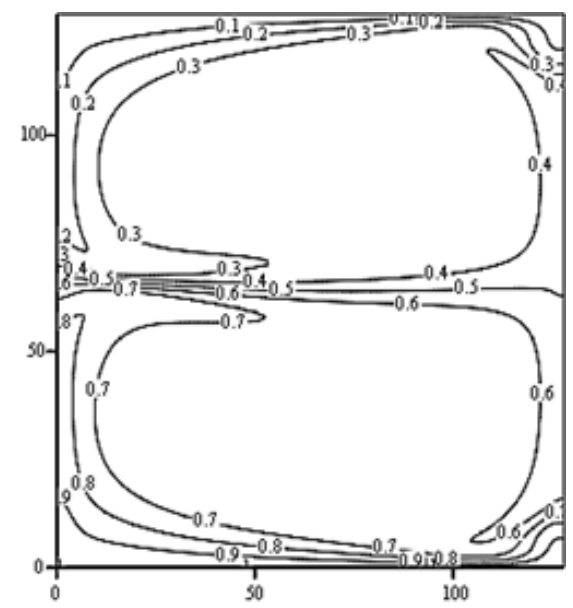

Fig. 7. Temperature contours for (a) Newtonian, (b) pseudoplastic fluids and (c) pseudoplastic fluids under the magnetic field effect at $R a=10^{6}$, respectively

For all fluids considered, as shown in Fig. 2, it has found one cell filled whole cavity at $R a=10^{4}$. The corresponding temperature profiles are shown in Fig. 3. For Newtonian, pseudoplastic fluids without any magnetic field effect and pseudoplastic fluids under the magnetic field effect, warm fluid rises near the left wall with the colder fluid falling near the opposite wall. As shown in Fig. 4 , the Newtonian fluid solution consists of two counter rotating cells and they are not symmetric; one vortex dominates the other vortex, whereas for pseudoplastic fluid without any magnetic field effect and pseudoplastic fluids under the magnetic field effect, we have two mirror image counter rotating the cells. Fig. 5 indicates that it is almost symmetric horizontally and warm fluid rises near the bottom while colder fluid is located near the top wall. As shown in Fig. 6, for Newtonian, pseudoplastic fluids without any magnetic field effect and pseudoplastic fluids under the magnetic field effect, streamline contours have two mirror image counter rotating cells. And finally in Fig. 7, temperature distribution is dense only near the wall and temperature contours are symmetric. Consequently, when $R a$ increases, streamline and temperature contours consist of two counter rotating cells, which are symmetric. As shown in all the figures, for pseudoplastic fluids, under the magnetic field effect, the intensity of the streamlines is decreased.

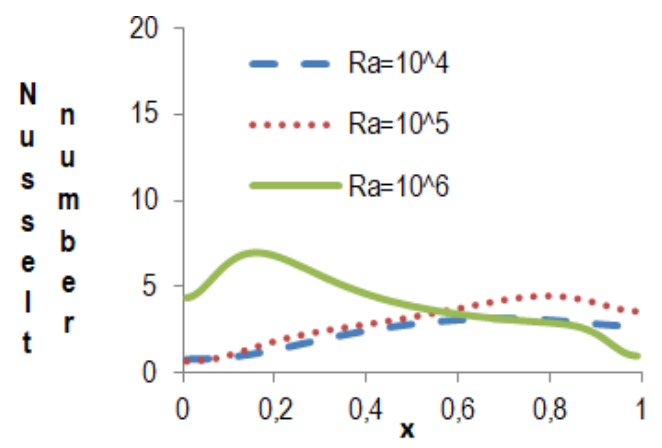

Fig. 8. Effect of local Nusselt number for Newtonian fluids

Fig. 8, 9 and 10 are respectively the plots of the local Nusselt number $(\mathrm{Nu})$ evaluated along the bottom wall for Newtonian, pseudoplastic fluids without any magnetic field effect and pseudoplastic fluids under the magnetic field effect. As shown in Fig. 8, for $R a=10^{4}$ and $R a=10^{5}$, the local Nusselt number has high values on the right wall, but it has low values for $R a=10^{6}$ on the same wall. In Fig. 9, for $R a=10^{4}$, the local Nusselt number has increased, but for $R a=10^{5}$, it has decreased, and on the right wall, there is almost no convective heat transfer. For $R a=10^{6}$, the local Nusselt number has reached up to a maximum level, and on the right and left walls, there is low convective heat transfer. Finally, in Fig. 10, the local Nusselt number results under the magnetic field effect are similar to the results obtained for Newtonian fluids without any magnetic field effect. As seen in Fig. 10, the magnetic field has made some effects for only $R a=10^{6}$.

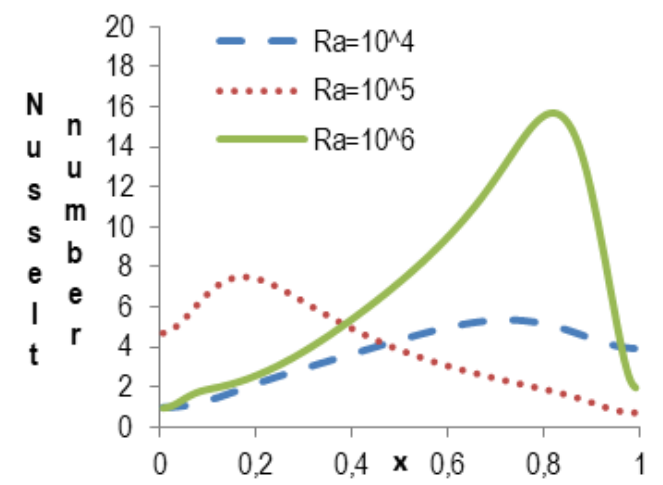

Fig. 9. Effect of local Nusselt number for pseudoplastic fluids without any magnetic field effect

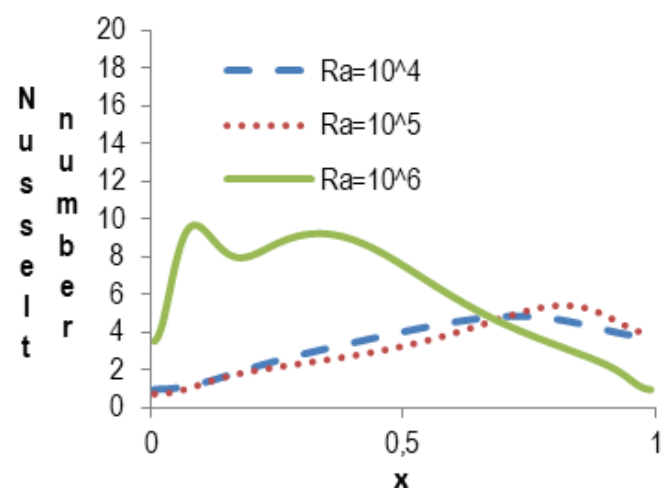

Fig. 10. Effect of local Nusselt number for pseudoplastic fluids under the magnetic field effect 


\section{CONCLUSIONS}

This paper mainly consisted of the computer simulation and the analysis of the streamline and temperature structures of $2 \mathrm{D}$ steady-state, incompressible fluid flow in an enclosed cavity region heated from below under the magnetic field effect. For Rayleigh number up to $R a=10^{6}$ and Chandrasekhar number up to 10 , the results have been presented and documented for the first time. The flow equations in stream function, vorticity and temperature formulation for pseudoplastic fluids were solved computationally using the numerical method described. In this numerical approach, the stream function, vorticity and energy equations were solved separately by using pseudo time derivative. For each equation, the numerical formulation required the solution of two tridiagonal systems, which allowed the use of large grid meshes easily and we used a fine grid mesh of $128 \times 128$. The method proved to be very effective on flow problems that required high accuracy on very fine grid meshes (Ertruk and Corke, 2001; Ertruk et al., 2004).

Our computations showed that fine grid mesh was necessary in order to obtain a steady solution, and when $R a$ and $\mathrm{Q}$ increased, streamline and temperature contours consisted of two counter rotating cells, which were symmetric. As a result, we obtained that when Rayleigh number is bigger than $10^{6}$ and Chandrasekhar number is bigger than 10 , instability occurs in the flow domain.

\section{REFERENCES}

1. Amber I., O'Donovan T. S. (2017), A numerical simulation of heat transfer in an enclosure with a nonlinear heat source, Numerical Heat Transfer, Part A: Applications, 71(11), 1081-1093.

2. Batchelor G. K. (1956), Steady laminar flow with closed streamlines at large Reynolds number, J. Fluid Mech., 1, 177-190.

3. Benjamin A. S., Denny V. E. (1979), On the convergence of numerical solutions for 2-D flows in a cavity at large Re, J. Comp. Physics, 33, 340-358.

4. De Vahl Davis G. (1983), Natural convection of air in a square cavity: A bench mark numerical solution, Int. J. for Num. Meth. in Fluids, 3, 249-264.

5. Demir H. (2005), Numerical modeling of viscoelastic cavity driven flow using finite difference simulations, Appl. Math. and Comp., 166, 64-83.

6. Elder J. W. (1965), Laminar free convection in a vertical slot, J. Fluid Mech., 23, 77-98.

7. Emery A., Chi H., Dale J. (1971), Free convection through vertical plane layers of non-Newtonian power law fluids, ASME J. Heat Transfer, 93, 164-171.

8. Erturk E., Corke T. C. (2001), Boundary layer leading-edge receptivity to sound at incidence angles, Journal of Fluid Mechanics, 444, 383-407.

9. Erturk E., Corke T. C., Gökçöl C. (2005), Numerical solutions of 2-D steady incompressible driven cavity flow at high Reynolds numbers, J. Numer. Meth. Fluids, 48, 747-774.

10. Erturk E., Haddad O. M., Corke T. C. (2004), Laminar incompressible flow past parabolic bodies at angles of attack, AIAA Journal, 42, 2254-2265.

11. Gebhart B., Jaluria Y., Mahajan R. L., Sammakia B. (1988), Buoyancy induced flows and transport, Washington: Hemisphere.

12. Ghia U., Ghia K. N., Shin C. T. (1982), High-Re solutions for incompressible flow using the Navier-Stokes equations and a multigrid method, J. Comp. Physics, 48, 387-411.
13. Gunzburger M. D., Meir A. J., Peterson J. S. (1991), On the existence, uniqueness and finite element approximation of solutions of the equations of stationary, incompressible magnetohydro-dynamics, Math. Comput., 56, 523-563.

14. Hasler U., Schneebeli A., Schötzau D. (2004), Mixed finite element approximation of incompressible MHD problems based on weighted regularization, Appl. Numer. Math., 51, 19-45.

15. He Y. N., Li, J. (2009), Convergence of three iterative methods based on the finite element discretization for the stationary NavierStokes equations, Comput. Methods Appl. Mech. Engrg., 198, 1351-1359.

16. Hou S., Zou Q., Chen S., Doolen G., Cogley A.C. (1995), Simulation of cavity flow by the Lattice Boltzmann method, J. Comp. Physics, 118, 329-347.

17. Khader M. M. (2016), Shifted Legendre Collocation method for the flow and heat transfer due to a stretching sheet embedded in a porous medium with variable thickness, variable thermal conductivity and thermal radiation, Mediterr. J. Math., 13, 2319-2336.

18. Liao S. J., Zhu J. M. (1996), A short note on higher-order stream function-vorticity formulation of 2-D steady state Navier-Stokes equations, Int. J. Numer. Methods Fluids, 22, 1-9.

19. Rayleigh R. (1916), On convection currents in a horizontal layer of fluid, when the higher temperature is on the underside, Phil. Mag., Ser.6, 32, 529-546.

20. Rubin S. G., Khosla P. K. (1981), N-S calculations with a coupled strongly implicit method, Computers and Fluids, 9, 163-180.

21. Rudraiah N., Barron R. M., Venkatachalappa M., Subbaraya C. K. (1995), Effect of a magnetic field on free convection in a rectangular enclosure, Int. J. Engng Sci., 33, 1075-1084.

22. Salah N. B., Soulaimani A., Habashi W. G. (2001), A finite element method for magnetohydrodynamics, Comput. Methods Appl. Mech. Engrg., 190, 5867-5892.

23. Schreiber R., Keller H. B. (1983), Driven cavity flows by efficient numerical techniques, J. Comp. Physics, 49, 310-333.

24. Shenoy A. V. (1988), Natural convection heat transfer to viscoelastic fluids, Houston: Gulf.

25. Siddheshwar P. G., Ramachandramurthy V., Uma D. (2011), Rayleigh-Benard and Marangoni magnetoconvection in Newtonian liquid with thermorheological effects, Int. J. Engng Sci., 49, 1078-1094.

26. Siginer D. A., Valenzuela-Rendon A. (1994), Natural convection of viscoelastic liquids, Proc. ASME Fluids Engineering Division Summer Meeting, Symposium, ASME FED, 179, 31-41.

27. Smith G. D. (1978), Numerical solution of partial differential equations by finite difference methods, Oxford University Pres.

28. Tennehill J. C., Anderson D. A., Pletcher R. H. (1997), Computational fluid mechanics and heat transfer, Taylor\& Francis.

29. Venkatachalappa M., Younghae D., Sankar M. (2011), Effect of magnetic field on the heat and mass transfer in a vertical annulus, Int. J. Engng Sci., 49, 262-278.

30. Wilkes J. O., Churehill S. W. (1966), The finite-difference computation of natural convection in a rectangular enclosure, AICHEJ, 12, 161-166.

31. Xu H., He Y. N. (2013), Some iterative finite element methods for steady Navier-Stokes equations with different viscosities, J. Comput. Phys., 232, 136-152. 